\title{
On transient stability of multi-machine power systems through Takagi-Sugeno fuzzy-based sliding mode control approach
}

\author{
E. Sharifi ${ }^{1}$ - A. H. Mazinan ${ }^{1}$
}

Received: 1 October 2016 / Accepted: 1 November 2017 / Published online: 20 November 2017

(C) The Author(s) 2017. This article is an open access publication

\begin{abstract}
The present research focuses on transient stability of multi-machine power systems in a full consideration regarding the performances of the Takagi-Sugeno fuzzybased sliding mode control approach in association with the conventional sliding mode and also the optimal control approaches to improve the last finding outcomes in this area. Hereinafter, concerning the robustness of the sliding mode control approach toward parametric uncertainties and environment perturbations, in fact, a couple of different sliding mode control approaches are designed for mutual comparison, after a number of state-of-the-art technique considerations. To increase the control performance, the Takagi-Sugeno fuzzy-based approach is devised to provide the appropriate coefficients. Finally, the three control approaches are all carried out in the six-machine power system under the same condition and the investigated results are correspondingly provided to be analyzed. The results indicate that the proposed fuzzy-based control approach is well behaved with respect to other related ones.
\end{abstract}

Keywords Transient stability · Six-machine power system · Takagi-Sugeno fuzzy-based sliding mode control approach . Optimal control approach

A. H. Mazinan

mazinan@azad.ac.ir; ahmazinan@gmail.com;

ah_mazinan@yahoo.com

1 Department of Control Engineering, Faculty of Electrical Engineering, South Tehran Branch, Islamic Azad University, (IAU), No. 209, North Iranshahr St., P.O. Box 11365/4435, Tehran, Iran

\section{List of symbols}

$H \quad$ Inertial constant (s)

$M \quad$ Inertial coefficient (s)

$D \quad$ Convergence coefficient (pu)

$T_{S} \quad$ Time constant of input control system (s)

$T_{g} \quad$ Servo-motor time constant (s)

$P_{e} \quad$ Electrical power (pu)

$P_{m} \quad$ Mechanical power (pu)

$\omega \quad$ Angular speed (rad/s)

$\delta \quad$ Rotor angle ( $\mathrm{rad})$

$E_{q}^{\prime} \quad$ Internal transient voltage (pu)

$Y_{i j} \quad i-j$ line transmission admittance $(\mathrm{pu})$

\section{Introduction}

Transient stability is a concept to be considered for the purpose of measuring the performance of synchronous machines, which has the highest importance for longdistance grids. From a physical point of view, transient stability can be defined as the ability of a system to remain with synchronous outcomes during the occurrence of large perturbations. On its own, in fact, the stability is addressed as the property of a power system that enables it to maintain a stable equilibrium and return to an acceptable state, when faced with large perturbation for normal performance situations. A large number of investigations are dedicated to the types of stability of the power systems, where some of them have directly focused on the transient stability, in-depth. With this goal, a set of potential related works in this area are now listed. 


\section{Related works}

State-of-the-art investigations in the area of transient stability with its specific application to synchronous machines in a wide range of structure variations have been recently proposed. In one such case, Ashraf et al. have explored a Takagi-Sugeno fuzzy-based control approach in dealing with transient stability augmentation of multi-machine power system, while Bakhshi et al. have considered fuzzy-based damping control approach though local measurements for the purpose of enhancing transient stability in power systems $[1,2]$.

Schaab et al. have considered robust control for voltage and transient stability of power grids focusing on wind power, and also Mazhari et al. have addressed a frequency-domain approach for distributed harmonic analysis in multi-area interconnected power systems [3,4]. Darabian et al. have presented a power control strategy, to improve power system stability in the presence of wind farms by designing predictive control and Shah et al. have studied the performance improvement of intrusion detection with fusion of multiple sensors, while Wuthishuwong et al. have focused on consensus-based local information coordination for the networked control of the autonomous intersection management [5-7]. In Yipeng et al's. works, an integrated high side voltage control approach is presented to improve short-term voltage stability regarding the receiving-end power systems, while in Yan et al.'s research work, trajectory sensitivity analysis on the equivalent one-machine-infinite-bus in case of multi-machine systems for preventive transient stability control is researched $[8,9]$.

In Godpromesse et al's. research, online simplified nonlinear control approach for transient stabilization enhancement of multi-machine power systems is considered, whereas in Haotian et al.'s research, switching excitation control approach for enhancement of transient stability of such systems is investigated [10,11]. Jiebei et al.'s research is to deal with generic inertia emulation controller for multi-terminal voltage-source-converter high voltage direct current systems. Shahgholian et al.'s research copes with power system stabilizer and flexible alternating current transmission systems control approach coordinated design via adaptive velocity update relaxation particle swarm optimization algorithm. Hui et al.'s research handles Lyapunov-based decentralized excitation control for global asymptotic stability and voltage regulation of the same multi-machine power systems, and subsequently Hongshan et al.'s investigation designs excitation prediction control in case of the aforementioned multi-machine power systems through balanced reduced model [10-14]. Shi et al. have proposed stabilizing control with transmission losses based on the pseudo-generalized Hamiltonian theory. Agrawal et al. have addressed support vector clustering-based direct coherency identification of generators and Ningqiang et al. have described damping Torques during transient behaviors as well [15-18]. Du et al.'s work considers robustness of an energy storage systembased stabilizer to suppress inter-area oscillations. Shojaeian et al.'s work explores damping of low-frequency oscillations in case of multi-machine power systems, based on adaptive input-output feedback linearization control. Sheng-Kuan et al.'s work realizes the objective function and algorithm for optimal design. Son et al.'s study is on the direct stability analysis, and finally Muyeen et al.'s work explains the reduction of frequency fluctuation for wind farm-connected power systems by an adaptive artificial neural network controlled energy capacitor system [19-23]. Seung-Ju et al.'s research discusses the passivity-based output synchronization of portcontrolled Hamiltonian and general linear interconnected systems, and Casagrande et al.'s work describes a solution to the multi-machine transient stability problem and simulated validation in realistic scenarios $[24,25]$.

Thereafter, Dragosavac et al. have proposed practical implementation of coordinated control, and Qiqi et al. have investigated the power angle control in case of gridconnected doubly fed induction generator wind turbines for fault ride-through, while Chaudhuri et al. have addressed system frequency support via multi-terminal direct current grids [26-28]. In Bijami et al.'s research, stabilizing signals for power system damping using generalized predictive control is designed through a new hybrid shuffled frog leaping algorithm, while in Chun-Feng et al.'s research, the coordinated control of flexible AC transmission system devices via an evolutionary fuzzy lead-lag control approach is realized under advanced continuous ant colony optimization [29,30]. In Wang et al.'s investigations, a number of aspects of stability enhancement based on offshore wind farm fed are addressed to deal with a multi-machine system $[31,32]$. Finally, in Yija et al.'s research, a nonlinear variable structure stabilizer in power system stability is discussed. In Qiang et al.'s research, nonlinear stabilizing control in the aforementioned multi-machine systems is described [32-34].

The rest of the paper is organized as follows: the proposed control approaches are given in Sect. 3, where the simulation results are all illustrated in Sect. 1. The research concludes the investigated outcomes in Sect. 2.

\section{The proposed control approaches}

\section{The preliminary information}

At first, to propose the control approaches, in an efficient manner, the multi-machine system with $m+1$ machines needs to be considered, though its differential equations in case of the ith machine to be rapidly simulated and the effect of poles can be highlighted. 


$$
\left\{\begin{array}{l}
\dot{P}_{m i}=\frac{1}{T_{d o i}}\left(-P_{m i}+P_{m i 0}\right)+\frac{1}{T_{d o i}^{\prime}} u_{i} \\
\dot{w}_{i}=\frac{w_{0}}{M_{i}}\left(P_{m i}-\frac{D_{i}}{w_{0}} w_{i}-\sum_{\substack{j=1 \\
i \neq j}}^{n+1} E_{q i}^{\prime} E_{q j}^{\prime} Y_{i j} \sin \left(\delta_{i}-\delta_{j}-\theta_{i j}\right)\right.
\end{array}\right)
$$

where $\delta_{i}$ is the angle, $w_{i}$ is the angular velocity, $P_{m i}$ is the input mechanical power, $E_{q i}^{\prime}$ is the component $q$ of voltage of transmission reactance, $M_{i}$ is the moment inertia, $D_{i}$ is the mechanical damping, $x_{d i}$ is the synchronous reactance of axis $d$ and $x_{d i}^{\prime}$ is the transient reactance of axis $d$ (all) in $i$ th machine. Additionally, $T_{d o i}^{\prime}, G_{i j}$ and $B_{i j}$ are the transient time constant of axis $d$, the transmission admittance between $i$ th and $j$ th machines and the transmission conductance between $i$ th and $j$ th machines, respectively. Finally, $\Delta x_{d i}=x_{d i}-x_{d i}^{\prime}$ and $\alpha_{i j}=\pi / 2-\arcsin \left(B_{i j} / Y_{i j}\right)$ are defined and $U_{i}$ is indicated to be the power control for $i$ th machine of $U$. Subsequently, the state variables to be chosen are taken as

$\left\{\begin{array}{l}x_{i 1}=P_{m i} \\ x_{i 2}=w_{i} \\ x_{i 3}=\delta_{i}\end{array}\right.$

Dynamic equations are now represented in the state space by Eq. (5)

$\dot{X}_{i}=f_{i}\left(X_{i}\right)+B_{i} U_{i}$,

where

$$
\left\{\begin{array}{l}
f_{i 1}(X)=\frac{1}{T_{d o i}}\left(-P_{m i}+P_{m i 0}\right) \\
f_{i 2}(X)=\frac{w_{0}}{M_{i}}\left(P_{m i}-\frac{D_{i}}{w_{0}} w_{i}-\sum_{\substack{j=1 \\
i \neq j}}^{n+1} E_{q i}^{\prime} E_{q j}^{\prime} Y_{i j} \sin \left(\delta_{i}-\delta_{j}-\theta_{i j}\right)\right) \\
f_{i 1}(X)=\frac{1}{T_{d o i}}\left(-P_{m i}+P_{m i 0}\right)
\end{array}\right.
$$

$$
B_{i}=\left[\begin{array}{l}
\frac{1}{T_{d o i}^{\prime}} \\
0 \\
0
\end{array}\right]
$$

Differentiating from Eq. (7) yields

$$
\left\{\begin{array}{rl}
\dot{w}_{i}= & \underbrace{\frac{w_{0}}{M_{i}}\left(P_{m i}-\frac{D_{i}}{w_{0}} w_{i}-P_{e}\right)}_{f_{i 2}(X)} \\
P_{e}= & \sum_{\substack{j=1 \\
i \neq j}}^{n+1} E_{q i}^{\prime} E_{q j}^{\prime} Y_{i j} \sin \left(\delta_{i}-\delta_{j}-\theta_{i j}\right) \\
\dot{P}_{e}= & \sum_{\substack{j=1 \\
i \neq j}}^{n+1} E_{q i}^{\prime} E_{q j}^{\prime} Y_{i j} \cos \left(\delta_{i}-\delta_{j}-\theta_{i j}\right)\left(w_{i}-w_{j}\right) \\
\ddot{w}_{i}= & \frac{w_{0}}{M_{i}}\left(\dot{P}_{m i}-\frac{D_{i}}{w_{0}} \dot{w}_{i}-\dot{P}_{e}\right) \\
= & \frac{w_{0}}{M_{i}}\left(\left(\frac{1}{T_{d o i}}\left(-P_{m i}+P_{m i 0}\right)+\frac{1}{T_{d o i}} u_{i}\right)\right. \\
& \left.-\frac{D_{i}}{w_{0}}\left(\frac{w_{0}}{M_{i}}\left(P_{m i}-\frac{D_{i}}{w_{0}} w_{i}-P_{e}\right)\right)-\dot{P}_{e}\right)
\end{array} .\right.
$$

As it can be seen, the first derivative of the angular speed yields to the linear relation between inputs and angular speed. Then, the control system is like Eq. (11):

$$
\left\{\begin{array}{l}
\ddot{w}_{i}=A_{i}(x)+B_{i}(x) u_{i} \\
A_{i}(x)=\frac{w_{0}}{M_{i}}\left(\left(\frac{1}{T_{d o i}}\left(-P_{m i}+P_{m i 0}\right)\right)-\frac{D_{i}}{w_{0}} \dot{w}_{i}-\dot{P}_{e}\right) . \\
B_{i}(x)=\frac{w_{0}}{M_{i}} \frac{1}{T_{d o i}} u_{i}
\end{array}\right.
$$

It should be noted that, to represent a comprehensive model of a generator, more than three differential equations are acquired in accordance with some sources, Eqs. (5)(7). However, the remaining equations are only constructive for short time constants, which are negligible in designing the stabilizer. Now, to evaluate the performance of the proposed control approach, two common controlling methods are applied to the system under consideration as its simulated results are correspondingly illustrated.

\section{The conventional sliding mode control approach}

The conventional sliding mode control approach is now considered, while some notations, used in this research, are in vector and also are based on the model presented in the previous section [27]. This is mainly similarly notated between the case study under control and the proposed approach that is designed.

$$
\left\{\begin{array}{l}
\dot{z}_{i 1}=z_{i 2} \\
\dot{z}_{i 2}=z_{i 3} \\
\dot{z}_{i 3}=A_{i}(x)+B_{i}(x) U_{i}
\end{array}\right.
$$

for which

$z_{i 1}=\delta_{i}$. 
Now, the results can be written as

$$
\left\{\begin{array}{l}
\dot{z}_{i 1}=z_{i 2}=w_{i} \\
\dot{z}_{i 2}=z_{i 3}=f_{2}(x) \\
\dot{z}_{i 3}=A_{i}(x)+B_{i}(x) U_{i}
\end{array} .\right.
$$

By choosing Eqs. (2)-(15), we can note that

$V_{i}=A_{i}(x)+B_{i}(x) U_{i}$.

Therefore, it is deduced that

$U_{i}=\frac{V_{i}-A_{i}(x)}{B_{i}(x)}$.

The linear system is now taken in the form of Eq. (17), i.e.,

$\dot{Z}_{i}=A Z_{i}+B V_{i}$

in which

$$
\left\{\begin{array}{l}
A=\left[\begin{array}{lll}
0 & 1 & 0 \\
0 & 0 & 1 \\
0 & 0 & 0
\end{array}\right] \\
B=\left[\begin{array}{l}
0 \\
0 \\
1
\end{array}\right]
\end{array} .\right.
$$

The relations are brought for a multi-variable sliding mode control approach in vector form as below, where the sliding surface of the system is presented by

$\sigma(z)=C^{T} Z$

Moreover, the following can be noted:

$V=-\sum_{i=1}^{m} K_{i} Z_{i}$

where

$K_{i}=\left\{\begin{array}{l}\alpha_{i} \sigma(z) Z_{i}>0 \\ \beta_{i} \sigma(z) Z_{i}<0\end{array}\right.$.

As a result, the variable structure control is designed for multi-machine power systems as in Eq. (22), i.e.,

$U_{i}=\frac{-\sum_{i=1}^{m} K_{i} Z_{i}-A_{i}(x)}{B_{i}(x)}$.

\section{The optimal control approach}

The optimal control approach is now considered by focusing on the nonlinear equation of the system under control, and the mapping should be used to transform the results into its linear form [28]. In the sequel, the investigated outcomes in the aforementioned linear form are given as in Eq. (23):

$\left\{\begin{array}{l}z_{i 1}=\dot{w}_{i} \\ z_{i 2}=w_{i} \\ z_{i 3}=\delta_{i}-\delta_{0}\end{array}\right.$

Now, the solution is to find a feedback like Eq. (24):

$U_{i}=\alpha(x)+\beta(x) v$,

for which $\alpha(x)$ and $\beta(x)$ are defined as in Eq. (25):

$\left\{\begin{array}{l}\alpha_{i}(x)=T_{d o i} \frac{D_{i}}{w_{0}} \dot{w}_{i}+P_{m i}-P_{m i 0}+T_{d o i} \dot{P}_{e} \\ \beta_{i}(x)=T_{d o i} \frac{M_{i}}{w_{0}}\end{array}\right.$

The linear form of the system used in this research is as below:

$\dot{z}_{i}=A z_{i}+B v$

which is given in the canonical form. As a result, the optimal solution of the nonlinear system takes the form of the linear quadratic regulator $(\mathrm{LQR})$ regarding the linear system. Therefore, the optimal control law for the linear system of Eq. (26) via the LQR laws is designed as in Eq. (27)

$v_{i}=-K_{1 i} z_{1 i}-K_{2 i} z_{2 i}-K_{3 i} z_{3 i}$

where $K_{1,2,3 i}$ are the coefficients of the feedback gain, realized by solving the rickety algebraic equations of the linear system. Subsequently, the control signal is defined as in Eq. (28):

$$
\begin{aligned}
u_{i}= & T_{d o i} \frac{D_{i}}{w_{0}} \dot{w}_{i}+P_{m i}-P_{m i 0}+T_{d o i} \dot{P}_{e} \\
& -T_{d o i} \frac{M_{i}}{w_{0}}\left(-K_{1 i} z_{1 i}-K_{2 i} z_{2 i}-K_{3 i} z_{3 i}\right) .
\end{aligned}
$$

\section{The Takagi-Sugeno fuzzy-based sliding-mode control approach}

The Takagi-Sugeno fuzzy-based sliding-mode control approach is now considered, whilst the sliding mode velocity of systems are chosen as their outputs. Now, it is possible to define the sliding surface that is necessary to reach the 
equilibrium point as follows:

$\left\{\begin{array}{l}e_{i}=w_{i}-w_{i d} \\ S_{i}(x)=\dot{e}_{i}+c_{i} e_{i}\end{array}\right.$.

To reach the signal in each level, the derivative of the sliding mode can be used that is equalized to be zero as in the following procedure:

$$
\left\{\begin{array}{l}
S_{i}(x)=\dot{e}_{i}+c_{i} e_{i} \Rightarrow \dot{S}_{i}(x)=\ddot{e}_{i}+c_{i} \dot{e}_{i} \\
\quad=0 \Rightarrow \ddot{w}_{i}-\ddot{w}_{i d}+c_{1} \dot{e}^{\bullet} 1=0 \\
\Rightarrow A_{i}(x)+B_{i}(x) u_{i}-\ddot{w}_{i d}+c_{1} \dot{e}^{\bullet} 1=0 \Rightarrow u_{i e q} \\
\quad=\frac{1}{B_{i}(x)}\left(\ddot{w}_{i d}-c_{1} \dot{e}{ }^{\prime} 1-A_{i}(x)\right)
\end{array}\right.
$$

The role of this signal is to maintain the states of system in its defined level. Finally, the control signal via Eq. (31) is provided as

$u=u_{i}+u_{e q}$

As it can be seen, the above-captioned control signal in the sliding mode control approach is composed of the reach signal on the surface. One of the main problems, initiated from switching to reach signal, is the chattering effect. A possible remedy might be using the Takagi-Sugeno fuzzy-based control approach to adapt the coefficients of the switching signal with its distance to the sliding surface. Regarding the closer states of the system, the coefficients need to be taken, in its small values, to decrease the chattering phenomenon of the system. It is possible to rewrite the control signal as in (32), in which the reach signal is replaced by the Takagi-Sugeno fuzzy-based approach:

$u=u_{\text {fuzzy }}+u_{\text {eq }}$.

The proposed idea in this procedure is the Takagi-Sugeno fuzzy-based integral sliding mode control approach, as to realize it the sliding surface is provided by the following:

$$
\left\{\begin{array}{l}
\sigma_{i}=w_{i}^{\prime}+c_{1 i}\left(w_{i}-2 \pi 50\right) \\
S_{i}=\sigma_{i}-z_{i} \\
z_{i}^{\prime}=-k_{1} \sigma_{i}
\end{array}\right.
$$

As formulated before, the control signal is designated in the form of Eq. (34):

$u_{i}=-\frac{1}{B(x)}\left(k \operatorname{sign}(s)+k_{1} \sigma_{i}\right)$.

Using the Takagi-Sugeno fuzzy-based system, the control effort with the reduced chattering becomes:

$u_{i}=u_{\mathrm{fuzzy}}+k_{1} \sigma_{i}$.
Table 1 The rule based realized in the Takagi-Sugeno fuzzy-based control approach

\begin{tabular}{llllll}
\hline$S \backslash S^{\prime}$ & IMf_1 & IMf_2 & IMf_3 & IMf_4 & IMf_5 \\
\hline IMf_1 & OMF_5 & OMF_4 & OMF_4 & OMF_2 & OMF_1 \\
IMf_2 & OMF_4 & OMF_4 & OMF_4 & OMF_2 & OMF_1 \\
IMf_3 & OMF_2 & OMF_2 & OMF_3 & OMF_2 & OMF_1 \\
IMf_4 & OMF_2 & OMF_2 & OMF_2 & OMF_2 & OMF_1 \\
IMf_5 & OMF_1 & OMF_1 & OMF_1 & OMF_1 & OMF_1 \\
\hline
\end{tabular}

The behavior of the proposed Takagi-Sugeno fuzzy-based control approach is based on the reach condition, which directly determines the control effort. The condition of reaching the surface in the sliding mode control approach is brought via Eq. (36).

$S S^{\prime}<0$

In fact, the condition of Eq. (36) indicates that the states, in each point of the space, can move to the surface.

Regarding the Takagi-Sugeno fuzzy-based control approach, the input membership functions; IMF, of two inputs, including the sliding surface and also their derivatives, are composed of the five behavioral types of the triangular that are equally given in the normalized span of $[-1,1]$ as follows:

IMf_1 : Very small, IMf_2 : Small, IMf_3 : Zero, IMf_4 : Large, IMf_5 : Very large.

Correspondingly, the output membership functions, OMF, are taken as the constant numbers that are all chosen in the span of $[-10,-5,0,5,10]$. Now, the fuzzy control rules are all tabulated in Table 1.

\section{The simulation results}

The simulation results are carried out through the three aforementioned control approaches including the conventional sliding mode control, Takagi-Sugeno fuzzy-based integral sliding mode control and finally the optimal control as are all illustrated via similar situations to be tangibly compared. First, the results of the present control approaches are considered and then their strength in omitting the large instabilities is correspondingly compared. For this reason, the six-machine power system to be controlled is presented in Fig. 1 [34].

\section{The outcomes of the proposed control approaches}

The investigated outcomes of the three proposed control approaches are simulated. In this section of the presentation of the proposed research, the angular velocities of all 
Fig. 1 The six-machine power system [34]

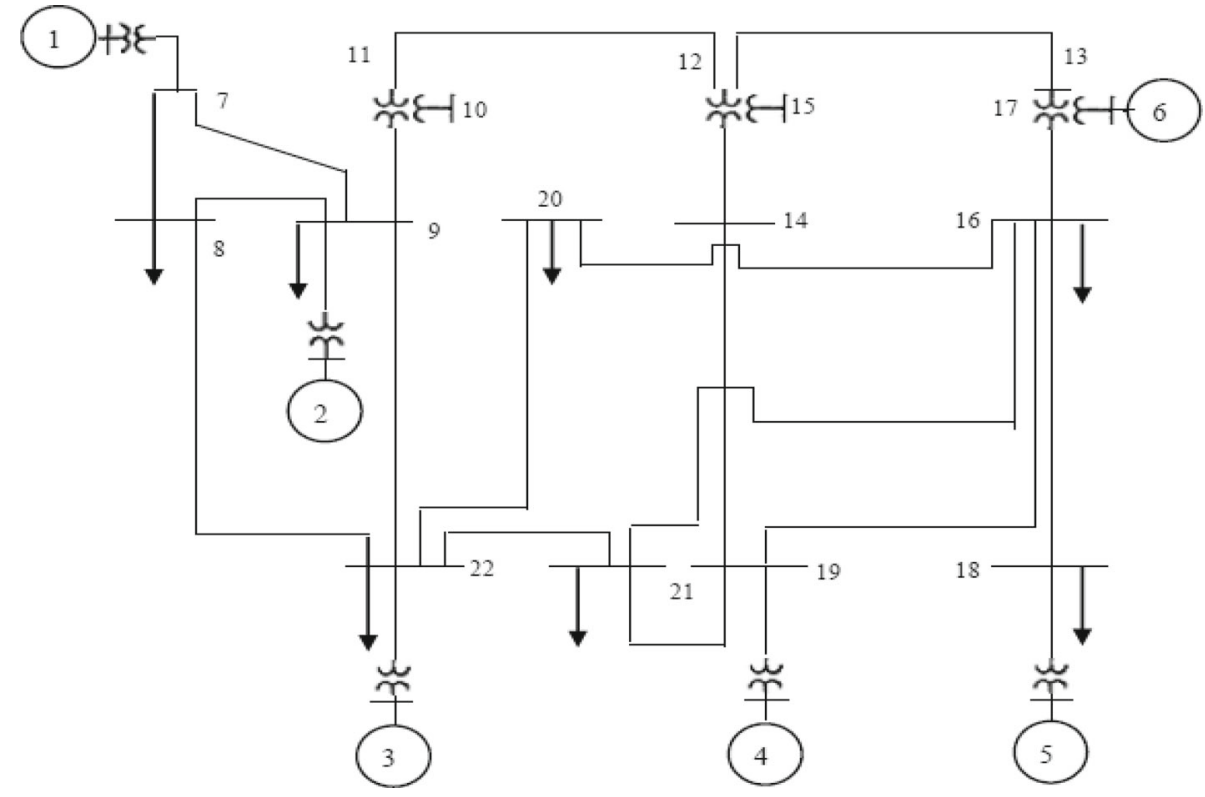

For this scheme, the power follows the desired value and is stabilized in nearly $10 \mathrm{~s}$, while the frequency of variations seems to be unacceptable. In other words, the main liability of the sliding mode control approach focusing on the chattering barely affects the output power. Hereinafter, the second control approach shows the better performance in time and accuracy, as its investigated outcomes are all illustrated in Fig. 3.

Subsequently, it is also shown that the Takagi-Sugeno fuzzy-based sliding mode control approach as the prominent proposed idea in this research shows a better performance under similar conditions in Fig. 4 by considering the rapid convergence and limiting its variations with respect to the conventional types of the control approaches. Here, the mechanical power is stabilized with the low frequency. The Takagi-Sugeno fuzzy-based control approach is accompanied by the integral sliding mode control to dampen the chattering significantly.

Although the acquired convergence rate investigated here is better than the optimal control approach, its rate of variations may be problematic. ried out by applying three control approaches and the results are correspondingly illustrated in the following figures. In the method, the formulation of the multi-machine power systems is brought from the potential materials. The first method is the optimal control approach, which transforms the nonlinear system to the LQR. The conventional sliding mode control approach, as the second method, and also the TakagiSugeno fuzzy-based control approach, as the third method, are all simulated. Both systems are used in similar conditions, i.e., the coefficients of the control and also the sliding surface are the same and it is tried to consider the effects of Takagi-Sugeno fuzzy-based control approach in a careful manner. Now, the mechanical power of the proposed first control approach is illustrated in Fig. 2.

\section{The outcomes of the proposed control approaches by focusing on the instantaneous perturbation}

The investigated outcomes of the proposed control approaches by focusing on the instantaneous perturbation are now considered, whilst the short circuit, the shutdown of the machine and so on can be taken into real consideration. They first occurred in $60 \mathrm{~s}$ of the simulations and the capabilities of the control approaches to overcome the problem were then evaluated. With this goal, the mechanical power of the system under control is stabilized due to the stability of the angular 


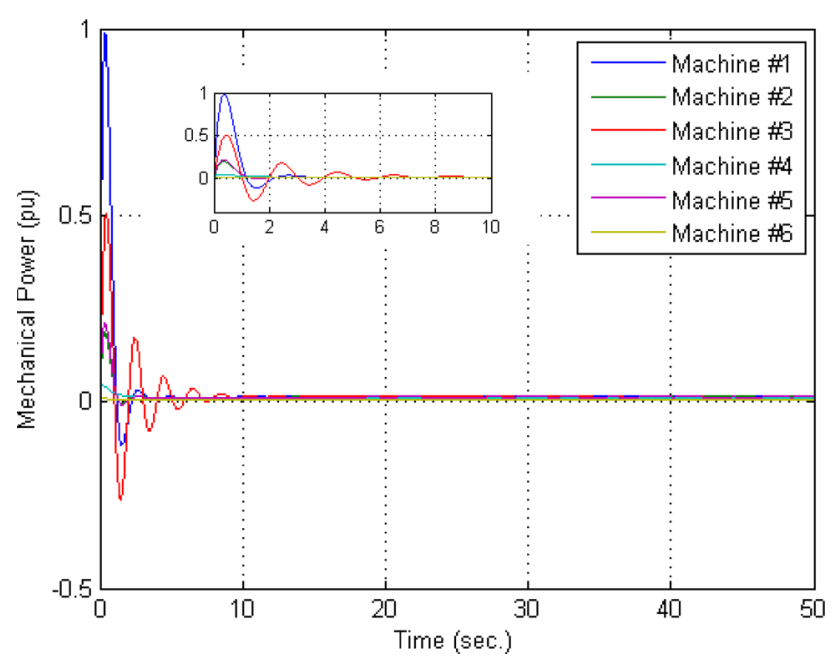

Fig. 3 The mechanical power in the second control approach

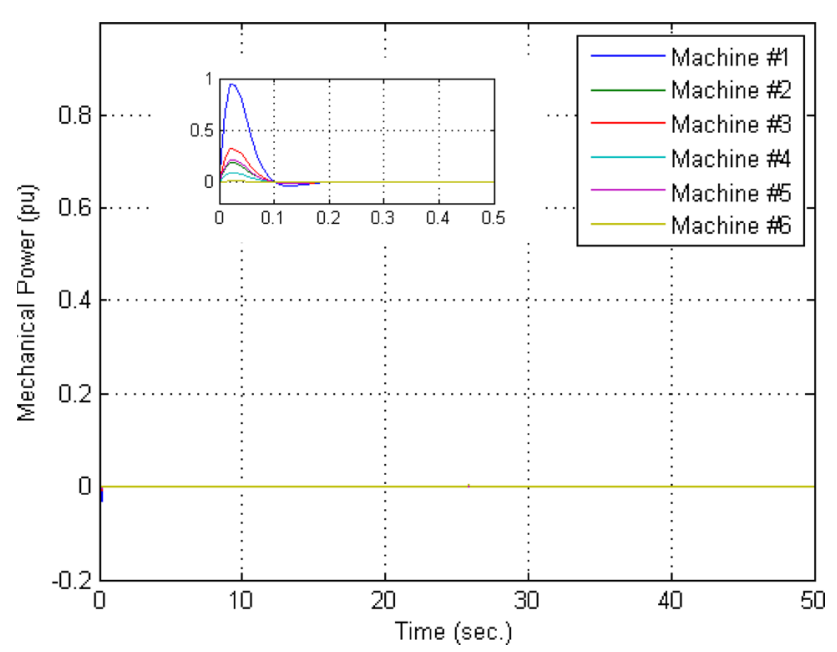

Fig. 4 The mechanical power in the third control approach

speed, as illustrated in Figs. 5, 6 and 7 for all of the proposed control approaches, respectively.

Now, note that by applying the perturbation to the system, the control approaches should try to regulate them and also to stabilize the aforementioned system. It is deduced through Fig. 6 that the proposed third control approach has a smaller settling time of about $1 \mathrm{~s}$ and lower oscillations as well. Also, Fig. 8 shows the differences between the performances of the control approaches in terms of one of the machines to be randomly chosen prior to and also after applying the perturbation.

As is obvious in the above-referenced Fig. 8, the outcomes of the proposed third control approach regarding the chosen machine\#6 indicate the best performance with respect to other related ones. On the other hand, the Takagi-Sugeno fuzzy-based control approach is well behaved to control the system in the presence of the perturbations. Note that the set of variations is significant and yields chattering in the

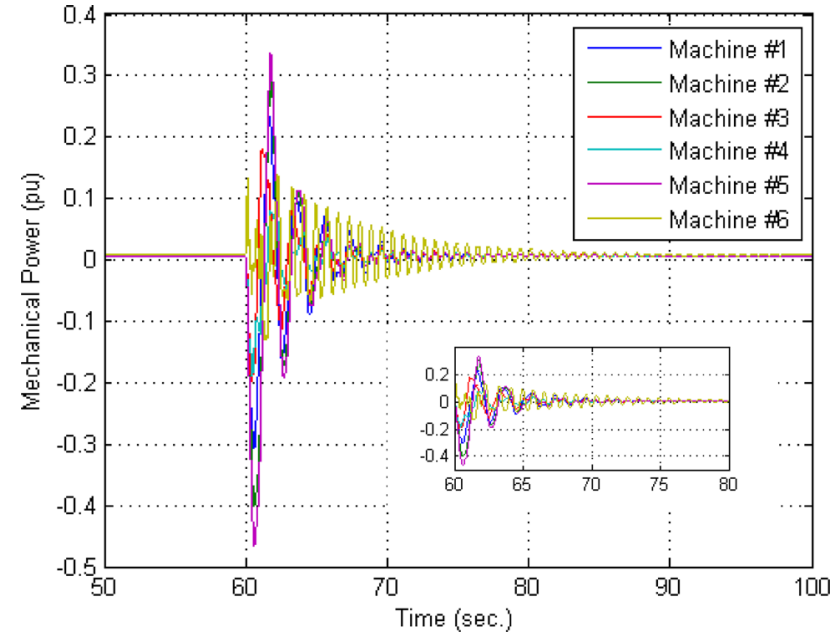

Fig. 5 The mechanical power in the first control approach

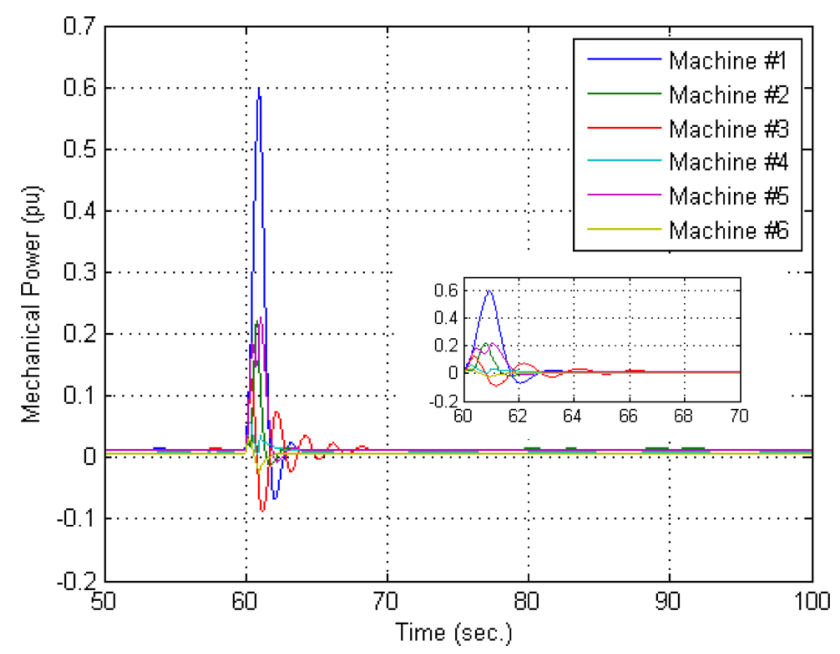

Fig. 6 The mechanical power in the second control approach

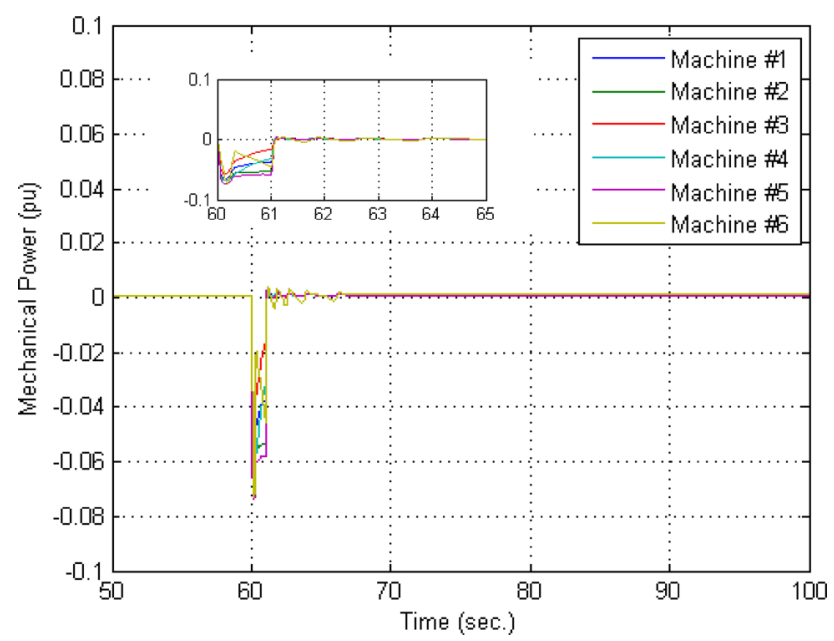

Fig. 7 The mechanical power in the third control approach 

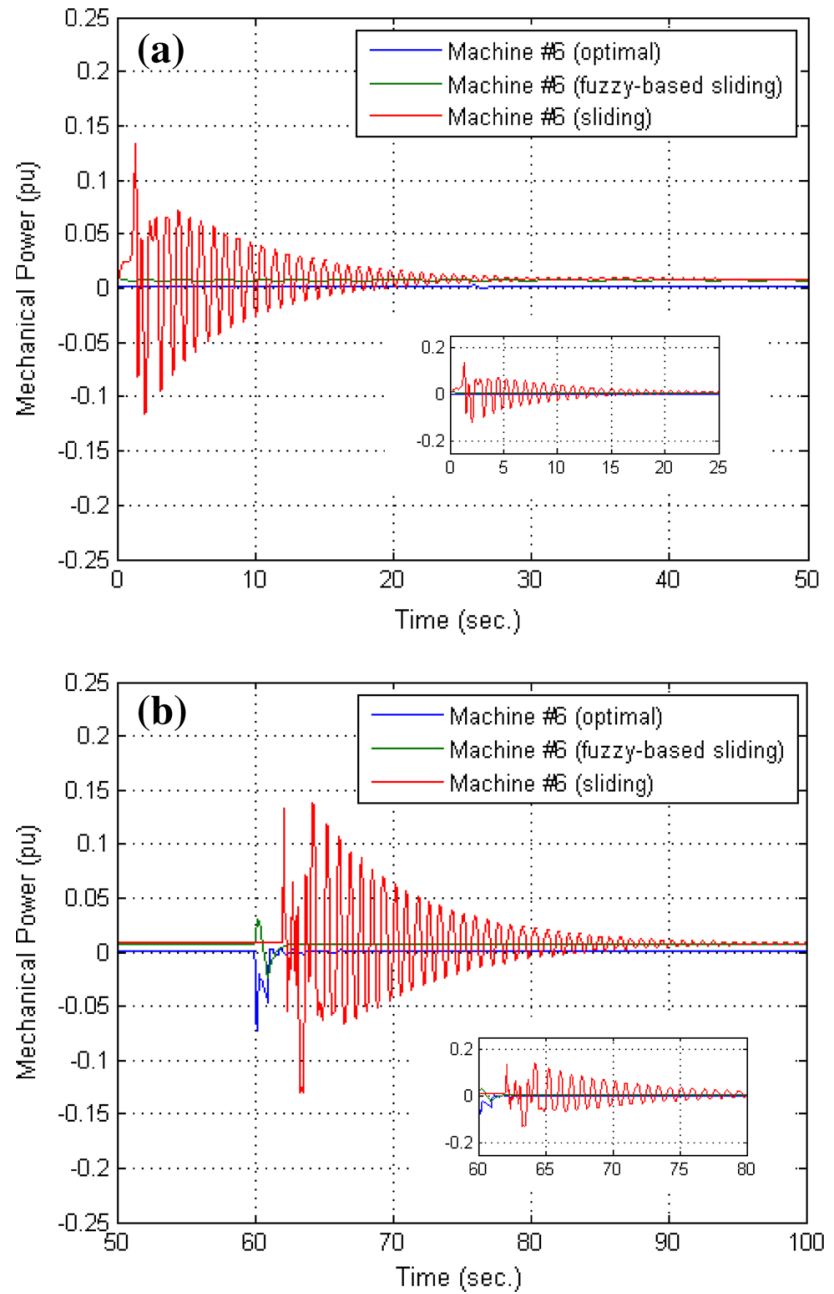

Fig. 8 The comparison of mechanical power in the three control approaches, a prior to applying perturbation, $\mathbf{b}$ after applying perturbation

Table 2 The performance comparison regarding the proposed control approaches in accordance with the settling time and the variation frequency

\begin{tabular}{lcl}
\hline The approaches & Settling time(s) & Variation frequency \\
\hline The optimal control & 10 & Medium \\
The sliding mode control & 5 & Severe \\
The Takagi-Sugeno & 1 & Benign \\
fuzzy-based control & & \\
\hline
\end{tabular}

control signal. The results of the second control approach indicate that there is no significant chattering and remains the stabilization to the large extent. In fact, by choosing a constant magnitude though the Takagi-Sugeno fuzzy-based control approach, it omits the chattering from the wind turbine. The performance comparison regarding the proposed control approaches in line with the settling time and the variation frequency is tabulated in Table 2 .

\section{Conclusion}

The six-machine power system is considered to be controlled through the conventional and Takagi-Sugeno fuzzy-based sliding mode control approaches, while the optimal control approach is also designed to have the merit of comparison. It is clearly concluded that the conventional one is not as efficient as the proposed Takagi-Sugeno fuzzy-based integral control approaches in general. As long as the same conventional control approach involves the high levels of the chattering on the sliding surface, the Takagi-Sugeno fuzzybased control approach tries to suppress the phenomenon. Besides, the results of the aforementioned Takagi-Sugeno fuzzy-based control approach are much quicker than other related considered ones. It is worth to noting that both the conventional and the Takagi-Sugeno fuzzy-based control approaches stabilize the system successfully after the occurrence of the grid faults to show acceptable efficiency. Stabilizing in a few seconds, the good accuracy and the ability to trace and follow the desired levels, prior to and after the fault, can be considered from the positive aspects of these control approaches. Although the higher control parameters in optimal control approach yields quicker stability, it fails to efficiently stabilize the system in the presence of perturbations. Regarding the supremacy of the robust control approach in overcoming the perturbations, it is shown that by realizing these types of control approaches in parallel, the effectiveness of the proposed Takagi-Sugeno fuzzy-based control approach is tangibly visible.

Open Access This article is distributed under the terms of the Creative Commons Attribution 4.0 International License (http://creativecomm ons.org/licenses/by/4.0/), which permits unrestricted use, distribution, and reproduction in any medium, provided you give appropriate credit to the original author(s) and the source, provide a link to the Creative Commons license, and indicate if changes were made.

\section{References}

1. Ashraf H, Mohd H (2015) A fuzzy logic controlled bridge type fault current limiter for transient stability augmentation of multimachine power system. IEEE Trans Power Syst 31:602-611

2. Bakhshi M, Holakooie MH, Rabiee A (2007) Fuzzy damping controller for TCSC using local measurements to enhance transient stability of power systems. Int J Electr Power Energy Syst 85:1221

3. Schaab K, Hahn J, Wolkov M, Stursberg O (2017) Robust control for voltage and transient stability of power grids relying on wind power. Control Eng Pract 60:7-17

4. Mazhari SM, Kouhsari SM, Ramirez A (2017) A novel frequencydomain approach for distributed harmonic analysis of multi-area interconnected power systems. Electr Power Syst Res 143:669-681

5. Darabian M, Jalilvand A (2017) A power control strategy to improve power system stability in the presence of wind farms using 
FACTS devices and predictive control. Int J Electr Power Energy Syst 85:50-66

6. Shah V, Aggarwal AK, Chaubey N (2017) Performance improvement of intrusion detection with fusion of multiple sensors. Complex Intell Syst 3(1):33-39

7. Wuthishuwong C, Traechtler A (2017) Consensus-based local information coordination for the networked control of the autonomous intersection management. Complex Intell Syst 3(1):17-32

8. Yipeng D, Xiaorong X, Baorong Z, Wenbo S (2015) An integrated high side var-voltage control strategy to improve short-term voltage stability of receiving-end power systems. IEEE Trans Power Syst 31:2105-2115

9. Yan X, Zhao Y, Junhua Z, Yusheng X (2015) Trajectory sensitivity analysis on the equivalent one-machine-infinite-bus of multi-machine systems for preventive transient stability control. IET Gener Transm Distrib 9:276-286

10. Godpromesse K, René F, Hilaire B (2015) An online simplified nonlinear controller for transient stabilization enhancement of DFIG in multi-machine power systems. IEEE Trans Autom Control 60:2464-2469

11. Haotian K, Yang L, Wu QH, Xiaoxin Z (2015) Switching excitation controller for enhancement of transient stability of multi-machine power systems. CSEE J Power Energy Syst 1:86-93

12. Jiebei Z, Josep M, William H, Campbell D (2014) Generic inertia emulation controller for multi-terminal voltage-source-converter high voltage direct current systems. IET Renew Power Gener 8:740-748

13. Shahgholian G, Movahedi A (2016) Power system stabilizer and flexible alternating current transmission systems controller coordinated design using adaptive velocity update relaxation particle swarm optimisation algorithm in multi-machine power system. IET Gener Transm Distrib 10:1860-1868

14. Hui L, Zechun H, Yonghua S (2013) Lyapunov-based decentralized excitation control for global asymptotic stability and voltage regulation of multi-machine power systems. IEEE Trans Power Syst 27(4):2262-2270

15. Hongshan Z, Xiaoming L, Ning X, Binbin W (2014) Excitation prediction control of multi-machine power systems using balanced reduced model. IET Gener Transm Distrib 8(6):1075-1081

16. Shi F, Wang J (2012) Stabilising control of multi-machine power systems with transmission losses based on pseudo-generalised Hamiltonian theory. IET Control Theory Appl 6(2):173-181

17. Agrawal R, Thukaram D (2013) Support vector clustering-based direct coherency identification of generators in a multi-machine power system. IET Gener Transm Distrib 7(12):1357-1366

18. Ningqiang J, Hsiao-Dong C (2014) Damping torques of multimachine power systems during transient behaviors. IEEE Trans Power Syst 29(3):1186-1193

19. Du W, Wang H, Cao J, Xiao L (2012) Robustness of an energy storage system-based stabilizer to suppress inter-area oscillations in a multi-machine power system. IET Gener Transm Distrib 6(4):339_ 351

20. Shojaeian S, Soltani J, Arab Markadeh G (2013) Damping of low frequency oscillations of multi-machine multi-UPFC power systems, based on adaptive input-output feedback linearization control. IEEE Trans Power Syst 27(4):1831-1840

21. Sheng-Kuan W (2013) A novel objective function and algorithm for optimal PSS parameter design in a multi-machine power system. IEEE Trans Power Syst 28(1):522-531
22. Son GT, Lee H-J, Lee S-Y, Park J-W (2012) A study on the direct stability analysis of multi-machine power system with resistive SFCL. IEEE Trans Appl Supercond 22(3):5602304. https://doi. org/10.1109/TASC.2011.2177626

23. Muyeen S, Hasanien H, Tamura J (2012) Reduction of frequency fluctuation for wind farm connected power systems by an adaptive artificial neural network controlled energy capacitor system. IET Renew Power Gener 6(4):226-235

24. Seung-Ju L, Kwang-Kyo O, Hyo-Sung A (2013) Passivity-based output synchronisation of port-controlled hamiltonian and general linear interconnected systems. IET Control Theory Appl 7(2):234245

25. Casagrande D, Astolfi A, Langarica D, Ortega R (2015) Solution to the multi-machine transient stability problem and simulated validation in realistic scenarios. IET Gener Trans Distrib 8(8):1392-1405

26. Dragosavac J, Janda Z, Milanovic J, Mihailovic J (2014) Practical implementation of coordinated Q-V control in a multi-machine power plant. IEEE Trans Power Syst 29(6):2883-2891

27. Qiqi BuS, Wenjuan D, Wang H, Gao S (2013) Power angle control of grid-connected doubly fed induction generator wind turbines for fault ride-through. IET Renew Power Gener 7(1):18-27

28. Chaudhuri N, Majumder R, Chaudhuri B (2013) System frequency support through multi-terminal DC (MTDC) grids. IEEE Trans Power Syst 28(1):347-356

29. Bijami E, Askari J, Farsangi M (2012) Design of stabilising signals for power system damping using generalised predictive control optimised by a new hybrid shuffled frog leaping algorithm. IET Gener Trans Distrib 6(10):1036-1045

30. Chun-Feng L, Hsu C, Juang C (2013) Coordinated control of flexible $\mathrm{AC}$ transmission system devices using an evolutionary fuzzy lead-lag controller with advanced continuous ant colony optimization. IEEE Trans Power Syst 28(1):385-392

31. Wang L, Truong D (2013) Stability enhancement of DFIG-based offshore wind farm fed to a multi-machine system using a STATCOM. IEEE Trans Power Syst 28(3):2882-2889

32. Wang L, Thi M (2013) Stability enhancement of a PMSG-based offshore wind farm fed to a multi-machine system through an LCCHVDC link. IEEE Trans Power Syst 28(3):3327-3334

33. Cao Y, Jiang L, Cheng S, Chen D, Malik OP, Hope GS (1994) A nonlinear variable structure stabilizer for power system stability. IEEE Trans Energy Convers 9(3):489-495

34. Lu Q, Sun YZ (1989) Nonlinear stabilizing control of multimachine systems. IEEE Trans Power Syst 4(1):236-241

Publisher's Note Springer Nature remains neutral with regard to jurisdictional claims in published maps and institutional affiliations. 